\title{
Hermite's Constant for Quadratic Number Fields
}

\author{
Ricardo Baeza, Renaud Coulangeon, Maria Ines Icaza, and Manuel O'Ryan
}

\section{CONTENTS}

1. Introduction

2. Bounds for Minimal Vectors of Humbert Forms

3. Examples

References
Baeza was partially supported by Fondecyt grant 1970214 and P. Formas cuadraticas, U. de Talca. Coulangeon was partially supported by European Union grant \#CI1*-CT93-0353. Icaza and O'Ryan were partially supported by Fondecyt grant 1990897 and P. Formas cuadraticas, U. de Talca.
We develop a method to compute the Hermite-Humbert constants $\gamma_{K, n}$ of a real quadratic number field $K$, the analogue of the classical Hermite constant $\gamma_{n}$ when $\mathbb{Q}$ is replaced by a quadratic extension. In the case $n=2$, the problem is equivalent to the determination of lowest points of fundamental domains in $\mathbb{H}^{2}$ for the Hilbert modular group over $\mathrm{K}$, that had been studied experimentally by $\mathrm{H}$. Cohn. We establish the results he conjectured for the fields $\mathbb{Q}(\sqrt{2}), \mathbb{Q}(\sqrt{3})$ and $\mathbb{Q}(\sqrt{5})$. The method relies on the characterization of extreme forms in terms of perfection and eutaxy given by the second author in an earlier paper.

\section{INTRODUCTION}

Let $K / \mathbb{Q}$ be a quadratic totally real number field, with ring of integers $\mathcal{O}_{K}$ and discriminant $\mathrm{d}_{K}$. Let $S=\left(S_{1}, S_{2}\right)$ be a binary Humbert form over $K$ [Baeza and Icaza 1997; Icaza 1997], i.e., $S_{1}, S_{2}$ are positive definite $2 \times 2$ real symmetric matrices. We denote by $\mathbf{P} \subset \mathbb{R}^{6}$ the space of such forms. The group $\mathrm{GL}\left(2, \mathcal{O}_{K}\right)$ of invertible $2 \times 2$ matrices with entries in the ring of integers $\mathcal{O}_{K}$ of $K$ acts on $\mathbf{P}$ : if $U \in \mathrm{GL}\left(2, \mathcal{O}_{K}\right)$ and $U^{(1)}, U^{(2)}$ denote the images of $U$ under the 2 distinct embeddings of $\operatorname{GL}\left(2, \mathcal{O}_{K}\right)$ in $\operatorname{GL}(2, \mathbb{R})$, then $S[U]=\left(S_{1}\left[U^{(1)}\right], S_{2}\left[U^{(2)}\right]\right)$, where $A[B]$ means $B^{t} A B$ whenever the product is defined. The set of forms $\left\{S[U]: U \in \mathrm{GL}\left(2, \mathcal{O}_{K}\right)\right\}$ is the equivalence class of $S$. If $u \in \mathcal{O}_{K}^{2}$, we define the value of $S$ at $u$ by $S[u]=S_{1}\left[u^{(1)}\right] S_{2}\left[u^{(2)}\right]$, and the minimum of $S$ is then

$$
m(S)=\min \left\{S[u]: 0 \neq u \in \mathcal{O}_{K}^{2}\right\} .
$$

Let $\operatorname{det} S=\operatorname{det} S_{1} \operatorname{det} S_{2}$. Then $\operatorname{det} S$ as well as $m(S)$ are class invariants of $S$, and we obtain the class-invariant function

$$
\gamma_{K}: \mathbf{P} \longrightarrow \mathbb{R}_{>0}, \quad \gamma_{K}(S)=\frac{m(S)}{(\operatorname{det} S)^{1 / 2}}
$$

This function is bounded by the constant $\left(\frac{\pi}{4}\right)^{2}\left|\mathrm{~d}_{K}\right|$ [Icaza 1997]. Actually Cohn has shown that $\frac{1}{2}\left|\mathrm{~d}_{K}\right|$ is a bound for $\gamma_{K}$ (see [Cohn 1965b] or [Ohno and 
Watanabe 2001] for a generalised version). Thus one can define the generalized Hermite constant of $K$ (for binary forms)

$$
\gamma_{K, 2}=\sup _{S \in \mathbf{P}} \gamma_{K}(S)
$$

The aim of this paper is to compute $\gamma_{K, 2}$ for the fields $\mathbb{Q}(\sqrt{2}), \mathbb{Q}(\sqrt{3})$ and $\mathbb{Q}(\sqrt{5})$, as well as to obtain general results towards the computation of $\gamma_{K, 2}$. This problem has been studied by H. Cohn [1965a; 1965b] under a different point of view. We now briefly relate $\gamma_{K, 2}$ to Cohn's work on lowest points of fundamental domains in $\mathbb{H}^{2}$ for the action of $\mathrm{SL}\left(2, \mathcal{O}_{K}\right)$, where $\mathbb{H}$ denotes the upper half plane $\{z \in \mathbb{C}: \operatorname{Im}(z)>0\}$.

If $\lambda=\left(\lambda_{1}, \lambda_{2}\right) \in \mathbb{R}_{>0} \times \mathbb{R}_{>0}$, it is clear that $\gamma_{K}(\lambda$. $S)=\gamma_{K}(S)$, where $\lambda \cdot S=\left(\lambda_{1} S_{1}, \lambda_{2} S_{2}\right)$. This invariance of $\gamma_{K}$ together with its class-invariance induces a function $\gamma_{K}: \mathbb{R}_{>0}^{2} \backslash \mathbf{P} / \mathrm{SL}\left(2, \mathcal{O}_{K}\right) \longrightarrow \mathbb{R}_{>0}$. Identifying $\mathbb{R}_{>0}^{2} \backslash \mathbf{P}$ with $\mathbf{S P}=\left\{\left(S_{1}, S_{2}\right) \in \mathbf{P}: \operatorname{det} S_{1}=\right.$ $\left.\operatorname{det} S_{2}=1\right\}$, we see that $\gamma_{K, 2}=\sup _{S \in \mathbf{S P}} m(S)$ and $\gamma_{K}: \mathbf{S P} \longrightarrow \mathbb{R}_{>0}$ is given by $\gamma_{K}=m(S)$. On the other hand, the group $\operatorname{SL}\left(2, \mathcal{O}_{K}\right)$ acts on $\mathbb{H}^{2}=\mathbb{H} \times \mathbb{H}$ by Möbius transformations by the rule

$$
\left(\begin{array}{cc}
\alpha & \beta \\
\gamma & \delta
\end{array}\right)\left(z_{1}, z_{2}\right)=\left(\frac{\alpha^{(1)} z_{1}+\beta^{(1)}}{\gamma^{(1)} z_{1}+\delta^{(1)}}, \frac{\alpha^{(2)} z_{2}+\beta^{(2)}}{\gamma^{(2)} z_{2}+\delta^{(2)}}\right)
$$

and we obtain a bijection $\varphi: \operatorname{SP} / \operatorname{SL}\left(2, \mathcal{O}_{K}\right) \longrightarrow$ $\mathbb{H}^{2} / \mathrm{SL}\left(2, \mathcal{O}_{K}\right)$ given by: for any $S=\left(S_{1}, S_{2}\right) \in \mathbf{S P}$ write $S_{j}=A_{j} A_{j}^{t}$ with $A_{j} \in \mathrm{SL}(2, \mathbb{R}), j=1,2$. Then $\varphi(S)$ is the class of $\left(A_{1}^{-1} i, A_{2}^{-1} i\right)$ in $\mathbb{H}^{2} / \mathrm{SL}\left(2, \mathcal{O}_{K}\right)$. More precisely, if

$$
S_{j}=\left(\begin{array}{ll}
a_{j} & b_{j} \\
b_{j} & d_{j}
\end{array}\right)
$$

for $j=1,2$, then

$$
\varphi(S)=\text { class of }\left(\frac{-b_{1}+i}{a_{1}}, \frac{-b_{2}+i}{a_{2}}\right) .
$$

Thus we have a function $\tilde{\gamma}_{K}: \mathbb{H}^{2} \longrightarrow \mathbb{R}_{>0}$ which on $\mathbb{H}^{2} / \mathrm{SL}\left(2, \mathcal{O}_{K}\right)$ is given by $\tilde{\gamma}_{K}=\gamma_{K} \circ \varphi^{-1}$. Now the relation of $\gamma_{K, 2}$ with the lowest points of fundamental domains in $\mathbb{H}^{2}$ is clear. We assert that under certain hypothesis on the field $K$ there exist fundamental domains $\mathbf{F} \subset \mathbb{H}^{2}$ for the action of $\operatorname{SL}\left(2, \mathcal{O}_{K}\right)$ such that

$$
\tilde{\gamma}_{K}\left(z_{1}, z_{2}\right)=\left(y_{1} y_{2}\right)^{-1}
$$

for all $\left(z_{1}, z_{2}\right) \in \mathbf{F}$, where $y_{j}=\operatorname{Im}\left(z_{j}\right)$ for $j=1,2$.
In particular,

$$
\gamma_{K, 2}=\left(\inf _{\left(z_{1}, z_{2}\right) \in \mathbf{F}}\left(y_{1} y_{2}\right)\right)^{-1}
$$

The classical case $K=\mathbb{Q}$ illustrates this formula nicely. Any reduced positive definite binary form $S=\left(\begin{array}{ll}a & b \\ b & c\end{array}\right)$ satisfies $0 \leq 2 b \leq a \leq c$ and $a=m(S)$. The corresponding point $\varphi(S)=z_{S} \in \mathbb{H}$ is $z_{S}=$ $-b / a+i / a \in \mathbf{F}=\left\{z \in \mathbb{H}:-\frac{1}{2} \leq x \leq \frac{1}{2},|z| \geq 1\right\}$. Thus $\operatorname{Im}\left(z_{S}\right)^{-1}=a=m(S)=\gamma_{\mathbb{Q}}(S)$ and we obtain $\gamma_{2}=\left(\inf _{z \in \mathbf{F}} y\right)^{-1}$. A closer look at $\mathbf{F}$ shows that $\inf _{z \in \mathbf{F}} y=\sqrt{3} / 2$, so that $\gamma_{2}=2 / \sqrt{3}$.

We now show assertion (1-3). We will assume that $K$ has class number 1 . Then any $S \in \mathbf{S P}$ is equivalent under $\operatorname{SL}\left(2, \mathcal{O}_{K}\right)$ to a form $S^{\prime}=S[U]$ such that

$$
m(S)=m\left(S^{\prime}\right)=S^{\prime}\left[(1,0)^{t}\right]=a_{1} a_{2} .
$$

This follows from the fact that all minimal vectors of $S$ are unimodular [Baeza and Icaza 1997] and that one can transform such vectors into $(1,0)$ by elements of $\operatorname{SL}\left(2, \mathcal{O}_{K}\right)$. Thus replacing $S$ by $S^{\prime}$ we may assume $m(S)=S\left[(1,0)^{t}\right]=a_{1} a_{2}$, where $S=$ $\left(\left(\begin{array}{ll}a_{1} & b_{1} \\ b_{1} & c_{1}\end{array}\right),\left(\begin{array}{ll}a_{2} & b_{2} \\ b_{2} & c_{2}\end{array}\right)\right)$. Now $z_{S}=\varphi(S) \in \mathbb{H}^{2}$ is given by

$$
\left(z_{1}, z_{2}\right)=\left(-\frac{b_{1}}{a_{1}}+\frac{1}{a_{1}} i,-\frac{b_{2}}{a_{2}}+\frac{1}{a_{2}} i\right)
$$

and hence

$$
\gamma_{K}(S)=m(S)=a_{1} a_{2}=\left(y_{1} y_{2}\right)^{-1} .
$$

This remark shows that we only need to look at fundamental domains in SP for $\operatorname{SL}\left(2, \mathcal{O}_{K}\right)$ where all elements $S$ have the property that $(1,0)$ is a minimal vector. Such fundamental domains can be constructed using Humbert's reduction theory [Humbert 1940]. The next result gives an explicit characterization of such domains.

For any $\alpha, \beta \in \mathcal{O}_{K}$ and $z=\left(z_{1}, z_{2}\right) \in \mathbb{H}^{2}$ let

$$
\mathrm{N}_{\alpha, \beta}(z)=\left|\alpha^{(1)} z_{1}+\beta^{(1)}\right|^{2}\left|\alpha^{(2)} z_{2}+\beta^{(2)}\right|^{2}
$$

and define

$$
\mathbf{F}_{0}=\bigcap_{\substack{\alpha, \beta \in \mathcal{O}_{K} \\\langle\alpha, \beta\rangle=\mathcal{O}_{K}}}\left\{z \in \mathbb{H}^{2}: \mathrm{N}_{\alpha, \beta}(z) \geq 1\right\},
$$

where $\langle\alpha, \beta\rangle$ is the ideal generated by $\alpha$ and $\beta$.

Proposition 1.1. Let $S \in \mathbf{S P}$ and let $z_{S} \in \mathbb{H}^{2}$ be the associated point in $\mathbb{H}^{2}$. The following assertions are equivalent:

1. $\tilde{\gamma}_{K}\left(z_{S}\right)=\left(y_{1} y_{2}\right)^{-1}$. 
2. $m(S)=S\left[(1,0)^{t}\right]$.

3. $z_{S} \in \mathbf{F}_{0}$.

Proof. The equivalence between statements 1 and 2 follows from the discussion above. Next assume 2, so that $S\left[(\alpha, \beta)^{t}\right] \geq S\left[(1,0)^{t}\right]$ for all $\alpha, \beta \in \mathcal{O}_{K}$ with $(\alpha, \beta) \neq(0,0)$. Writing $S=\left(\left(\begin{array}{ll}a_{1} & b_{1} \\ b_{1} & c_{1}\end{array}\right),\left(\begin{array}{ll}a_{2} & b_{2} \\ b_{2} & c_{2}\end{array}\right)\right)$, we get

$$
\begin{aligned}
& \prod_{j=1}^{2}\left(a_{j} \alpha^{(j) 2}+2 b_{j} \alpha^{(j)} \beta^{(j)}+c_{j} \beta^{(j) 2}\right) \geq a_{1} a_{2}, \\
& \prod_{j=1}^{2}\left(\alpha^{(j) 2}+2 \frac{b_{j}}{a_{j}} \alpha^{(j)} \beta^{(j)}+\frac{c_{j}}{a_{j}} \beta^{(j) 2}\right) \geq 1 .
\end{aligned}
$$

Since

$$
z_{S}=\left(-\frac{b_{1}}{a_{1}}+i \frac{1}{a_{1}},-\frac{b_{2}}{a_{2}}+i \frac{1}{a_{2}}\right),
$$

we have

$$
-\beta^{(j)} z_{j}+\alpha^{(j)}=\beta^{(j)} \frac{b_{j}}{a_{j}}+\alpha^{(j)}+\frac{\beta^{(j)}}{a_{j}} i
$$

and hence

$$
\begin{aligned}
\mathrm{N}_{-\beta, \alpha}\left(z_{S}\right) & \\
= & \left|-\beta^{(1)} z_{1}+\alpha^{(1)}\right|^{2}\left|-\beta^{(2)} z_{2}+\alpha^{(2)}\right|^{2} \\
& =\prod_{j=1}^{2}\left(\beta^{(j)^{2}}\left(\frac{b_{j}^{2}}{a_{j}^{2}}-\frac{1}{a_{j}^{2}}\right)+2 \frac{b_{j}}{a_{j}} \alpha^{(j)} \beta^{(j)}+\alpha^{(j) 2}\right) \\
& =\prod_{j=1}^{2}\left(\alpha^{(j) 2}+2 \frac{b_{j}}{a_{j}} \alpha^{(j)} \beta^{(j)}+\frac{c_{j}}{a_{j}} \beta^{(j) 2}\right) \geq 1,
\end{aligned}
$$

because $b_{j}^{2}-a_{j} c_{j}=1$ for $j=1,2$. This shows that $z_{S} \in \mathbf{F}_{0}$. Tracing back this computation, we see that statement 3 implies 2 .

Thus any fundamental domain $\mathbf{F}$ for $\operatorname{SL}\left(2, \mathcal{O}_{K}\right)$ in $\mathbb{H}^{2}$ contained in $\mathbf{F}_{0}$ satisfies $\gamma_{K, 2}=\left(\inf _{z \in \mathbf{F}} y_{1} y_{2}\right)^{-1}$, establishing the relationship between the search for lowest points in such domains [Cohn 1965a; 1965b] and the search for extreme Humbert forms. We will in the next section stick to this last point of view. Our results (Section 3) will explain some of Cohn's computations and guesses. In Section 2 we will estimate the absolute values of the components of minimal vectors of Humbert forms. This will enable us to find a finite set of forms where we can expect to find extreme forms. To find them in this set, one uses the analogue of Voronoi's theory for number fields developed in [Coulangeon 2001]. In Section 3 we apply this strategy to compute $\boldsymbol{\gamma}_{K, 2}$ for the fields $\mathbb{Q}(\sqrt{2}), \mathbb{Q}(\sqrt{3})$ and $\mathbb{Q}(\sqrt{5})$.

\section{BOUNDS FOR MINIMAL VECTORS OF HUMBERT FORMS}

Let $S \in \mathbf{P}$ be a binary Humbert form $\left(S_{1}, S_{2}\right)$. Recall that a vector $u \in \mathcal{O}_{K}^{2}$ is minimal if $S[u]=m(S)$. If $\varepsilon \in U_{k}$ is a unit of $K$, then $\varepsilon u$ is also a minimal vector of $S$ and the set $M(S)$ of classes of minimal vectors of $S$ is finite [Icaza 1997]. Moreover, as in the classical case, it is known that if $S$ is an extreme form, i.e., $\gamma_{K}(S)$ is a local maximum of $\gamma_{K}$, then the set $M(S)$ can not be too small. Using the characterization of extreme Humbert forms given in [Coulangeon 2001] we have:

Proposition 2.1. If $S$ is a perfect binary Humbert form over the totally real quadratic field $K$, then $|M(S)| \geq 5$.

Proof. Recall that a perfect Humbert form $S$ of rank $n$ over a (totally real) field $K$ of degree $r$ is characterized by the condition

$$
\operatorname{dim}_{\mathbb{R}} \sum_{u \in M(S)} \mathbb{R} u^{t} u=\frac{r n(n+1)}{2}-r+1,
$$

where $u^{t} u$ is the vector

$$
\left(\frac{u^{(1) t} u^{(1)}}{S_{1}\left[u^{(1)}\right]}, \ldots, \frac{u^{(r) t} u^{(r)}}{S_{r}\left[u^{(r)}\right]}\right)
$$

see [Coulangeon 2001]. Thus in our case we obtain $\operatorname{dim}_{\mathbb{R}} \sum_{u \in M(S)} \mathbb{R} u^{t} u=5$ and hence $|M(S)| \geq 5$.

Remark 2.2. In terms of the associated points $z_{S} \in$ $\mathbb{H}^{2}$, this result can be interpreted as follows: if $S \in$ SP is extreme, then $z_{S}$ lies at least on five hypersurfaces $\left\{\mathrm{N}_{\alpha, \beta}(z)=1\right\}$ on the boundary of $\mathbf{F}_{0}$.

Proposition 2.1 now suggests the following procedure to find extreme Humbert forms. First note that after scaling a form $S$ we can always assume $m(S)=1$. The strategy then is roughly as follows:

1. Find a finite set $M_{K} \subset \mathcal{O}_{K}^{2}$ such that any extreme binary Humbert form is equivalent under $\operatorname{GL}\left(2, \mathcal{O}_{K}\right)$ to a form $S$ with $\left|M(S) \cap M_{K}\right| \geq 5$.

2. For any 5-set $T=\left\{u_{1}, \ldots, u_{5}\right\} \subset M_{K}$ solve the linear equations in the unknown $S$

$$
S\left[u_{1}\right]=\cdots=S\left[u_{5}\right]=1 .
$$


3. Sort the resulting forms according to scaling $S \rightarrow$ $\lambda S, \lambda \in \mathbb{R}_{>0}^{2}$ and integal equivalence $S \rightarrow S[U]$, for $U \in \mathrm{SL}\left(2, \mathcal{O}_{K}\right)$.

4. Test for perfection and eutaxy of the remaining forms using [Coulangeon 2001].

Theoretically the existence of the set $M_{K}$ is assured by the reduction theory of P. Humbert [1940]. But unfortunately the description of the set of reduced Humbert forms involves unexplicit constants, which are difficult to estimate. The results in the next three lemmas will enable us to construct an explicit set $M_{K}$.

Lemma 2.3. Let $S=\left(S_{1}, S_{2}\right) \in \mathbf{P}$, with

$$
S_{i}=\left(\begin{array}{ll}
a_{i} & b_{i} \\
b_{i} & c_{i}
\end{array}\right)
$$

for $i=1,2$ and $u=(\alpha, \beta)^{t} \in \mathcal{O}_{K}^{2}$. Then

$$
\begin{aligned}
\left|\mathrm{N}_{K / \mathbb{Q}}(\alpha)\right| & \leq \sqrt{\frac{c_{1} c_{2}}{m(S)} \frac{S(u)}{m(S)}} \gamma_{K, 2}, \\
\left|\mathrm{~N}_{K / \mathbb{Q}}(\beta)\right| & \leq \sqrt{\frac{a_{1} a_{2}}{m(S)} \frac{S(u)}{m(S)}} \gamma_{K, 2}, \\
\left|\alpha^{(1)} \beta^{(2)}\right| & \leq \sqrt{\frac{a_{2} c_{1}}{m(S)} \frac{S(u)}{m(S)}} \gamma_{K, 2}, \\
\left|\alpha^{(2)} \beta^{(1)}\right| & \leq \sqrt{\frac{a_{1} c_{2}}{m(S)} \frac{S(u)}{m(S)}} \gamma_{K, 2} .
\end{aligned}
$$

Proof. We have

$$
\begin{aligned}
S_{i}\left[u^{(i)}\right] & =a_{i} \alpha^{(i)^{2}}+c_{i} \beta^{(i)^{2}}+2 b_{i} \alpha^{(i)} \beta^{(i)} \\
& =c_{i}\left(\beta^{(i)}+\frac{b_{i}}{c_{i}} \alpha^{(i)^{2}}+\frac{a_{i} c_{i}-b_{i}^{2}}{c_{i}} \alpha^{(i)^{2}}\right) \\
& \geq \frac{\left|S_{i}\right|}{c_{i}} \alpha^{(i)^{2}},
\end{aligned}
$$

and the same inequality holds replacing $\alpha$ by $\beta$, and $a_{i}$ by $c_{i}$ respectively. The conclusion follows, writing $S(u)=S_{1}\left[u^{(1)}\right] S_{2}\left[u^{(2)}\right]$ in all four possible ways, and using

$$
\frac{m(S)}{\sqrt{\left|S_{1}\right|\left|S_{2}\right|}} \leq \gamma_{K, 2}
$$

Lemma 2.4. Let $u={ }^{t}(\alpha, \beta)$ and $v={ }^{t}(\nu, \mu) \in \mathcal{O}_{K}^{2}$ be minimal vectors of $S$, with $v \notin \mathcal{O}_{K}^{\times} u$, and $U=\left(\begin{array}{c}\alpha \beta \\ \nu \mu\end{array}\right)$. Then

$$
\left|\mathrm{N}_{K / \mathbb{Q}}(\operatorname{det} U)\right| \leq \gamma_{K, 2}
$$

Proof. Consider the Humbert form $S[U]$. Clearly, $m(S[U])=m(S)$. On the other hand, $\operatorname{det} S[U]=$ $\mathrm{N}_{K / \mathbb{Q}}(\operatorname{det} U)^{2} \operatorname{det} S$, so that

$$
\mathrm{N}_{K / \mathbb{Q}}(\operatorname{det} U)^{2}=\frac{\operatorname{det} S[U]}{\operatorname{det} S} .
$$

Now

$$
\begin{aligned}
\operatorname{det} S[U] & =\operatorname{det} S_{1}\left[U^{(1)}\right] \operatorname{det} S_{2}\left[U^{(2)}\right] \\
& \leq\left(S_{1}\left[u^{(1)}\right] S_{1}\left[v^{(1)}\right]\right)\left(S_{2}\left[u^{(2)}\right] S_{2}\left[v^{(2)}\right]\right) \\
& =m(S)^{2}=\operatorname{det} S \gamma_{K}(S)^{2}
\end{aligned}
$$

Hence

$$
\mathrm{N}_{K / \mathbb{Q}}(\operatorname{det} U)^{2} \leq \gamma_{K}(S)^{2} \leq \gamma_{K, 2}^{2}
$$

As usual, we define the fundamental unit of $K$ as the uniquely determined fundamental unit $\varepsilon$ subject to the condition that $\varepsilon_{0}=\varepsilon^{(1)}>1$. We also define a constant $C_{K}$, depending only on $K$, as

$$
C_{K}=\sup _{\eta=(\alpha, \beta) \in K^{2}} \inf _{u=(x, y) \in \mathcal{O}_{K}^{2}}\|\eta-u\|_{\infty} .
$$

We also denote by $\left\{e_{1}, e_{2}\right\}$ the standard basis of $\mathcal{O}_{K}^{2}$.

Lemma 2.5. If $h(K)=1$, any binary Humbert form with at least 2 minimal vectors admits a representative $S=\left(S_{1}, S_{2}\right)$, modulo scaling and integral equivalence, such that

1. $m(S)=1, e_{1} \in M(S)$ and $S_{1}\left[e_{1}^{(1)}\right]=S_{2}\left[e_{1}^{(2)}\right]=1$, so that

$$
S=\left(\left(\begin{array}{cc}
1 & b_{1} \\
b_{1} & c_{1}
\end{array}\right),\left(\begin{array}{cc}
1 & b_{2} \\
b_{2} & c_{2}
\end{array}\right)\right)
$$

2. Any other minimal vector admits a representative $u=(x, y)^{t}$ modulo units satisfying

$$
\begin{aligned}
& \left|x^{(i)}\right|<\sqrt{\varepsilon_{0} \gamma_{K, 2}}\left(1+C_{K} \varepsilon_{0} \sqrt{\gamma_{K, 2}}\right), \\
& \left|y^{(i)}\right|<\varepsilon_{0}^{3 / 2} \gamma_{K, 2} .
\end{aligned}
$$

Proof. That one can assume that $e_{1}$ is minimal is clear since $h_{K}=1$ and any minimal vector is primitive, i.e., its coordinates are coprime. The condition $S_{1}\left[e_{1}^{(1)}\right]=S_{2}\left[e_{1}^{(2)}\right]=1$ is then easily fulfilled by scaling.

For the second assertion we proceed in several steps: first, let $u_{2}=\left(x_{2}, y_{2}\right)$, with $y_{2} \neq 0$, be a second minimal vector. We know from Lemma 2.4, applied to $U=\left(\begin{array}{ll}1 & x_{2} \\ 0 & y_{2}\end{array}\right)$, that $\left|\mathrm{N}_{K / \mathbb{Q}}\left(y_{2}\right)\right| \leq \gamma_{K, 2}$. Let $c_{i}^{\prime}=A_{i}\left[u_{2}^{(i)}\right]$, for $i=1,2$. Since $u_{2}$ is minimal and we are assuming that $m(S)=1$, we have $c_{1}^{\prime} c_{2}^{\prime}=1$. Multiplying $u_{2}$ by a unit $\eta=\varepsilon^{k}, k \in \mathbb{Z}$, 
changes $c_{1}^{\prime} / c_{2}^{\prime}$ into $\varepsilon_{0}^{4 k} c_{1}^{\prime} / c_{2}^{\prime}$, so we can assume that $\varepsilon_{0}^{-2} \leq c_{1}^{\prime} / c_{2}^{\prime}<\varepsilon_{0}^{2}$, whence

$$
\begin{aligned}
& \varepsilon_{0}^{-1} \leq c_{1}^{\prime}<\varepsilon_{0} \\
& \varepsilon_{0}^{-1}<c_{2}^{\prime} \leq \varepsilon_{0} .
\end{aligned}
$$

Let $Y=\left\{y \in \mathcal{O}_{K} \backslash\{0\}:\left|\mathrm{N}_{K / \mathbb{Q}}(y)\right| \leq \gamma_{K, 2}\right\}$, and define $\bar{Y}$ to be the set of $y \in Y$ such that $\sqrt{\varepsilon_{0}^{-1}} \leq$ $\left|y^{(1)}\right|<\sqrt{\varepsilon_{0} \gamma_{K, 2}}$ and $\sqrt{\varepsilon_{0}^{-1}}<\left|y^{(2)}\right| \leq \sqrt{\varepsilon_{0} \gamma_{K, 2}}$. This set is finite and any $y \in Y$ admits a representative modulo units in $\bar{Y}$. Thus, replacing $S$ by $S[U]$ with $U=\left(\begin{array}{ll}1 & 0 \\ 0 & \eta\end{array}\right)$, for a suitable unit $\eta$, one can assume that $y_{2}$ is in $\bar{Y}$ (indeed, replacing $S$ by $S[U]$ amounts to replace $e_{2}$ by $\eta e_{2}$, so $y_{2}$ is replaced by $\eta^{-1} y_{2}$, while $x_{2}$ remains unchanged).

We can moreover assume, replacing $S$ by $S\left[\left(\begin{array}{ll}1 & q \\ 0 & 1\end{array}\right)\right]$ for a suitable $q \in \mathcal{O}_{K}$, that

$$
\left|x_{2}^{(i)}\right| \leq C_{K} y_{2}^{(i)}, \quad i=1,2 .
$$

Finally, let $u=(x, y)^{t}$ be any minimal vector; we show that, up to units, its coordinates can be bounded according to the lemma. We claim that there exists $\lambda \in \mathcal{O}_{K}$ with $\left|\mathrm{N}_{K / \mathbb{Q}}(\lambda)\right| \leq \gamma_{K, 2}$ such that $\lambda u \in \mathcal{O}_{K} e_{1} \oplus \mathcal{O}_{K} u_{2}$, i.e.,

$$
u=\frac{\tilde{x}}{\lambda} e_{1}+\frac{\tilde{y}}{\lambda} u_{2}, \quad \tilde{x}, \tilde{y} \in \mathcal{O}_{K} .
$$

The set choice of such $\lambda_{\mathrm{s}}$ is stable under multiplication by units, so we can assume without loss of generality that

$$
\begin{aligned}
& \sqrt{\varepsilon_{0}^{-1}\left|\mathrm{~N}_{K / \mathbb{Q}}(\lambda)\right|} \leq\left|\lambda^{(1)}\right|<\sqrt{\varepsilon_{0}\left|\mathrm{~N}_{K / \mathbb{Q}}(\lambda)\right|}, \\
& \sqrt{\varepsilon_{0}^{-1}\left|\mathrm{~N}_{K / \mathbb{Q}}(\lambda)\right|}<\left|\lambda^{(2)}\right| \leq \sqrt{\varepsilon_{0}\left|\mathrm{~N}_{K / \mathbb{Q}}(\lambda)\right|},
\end{aligned}
$$

which allows a finite set of values for $\lambda$. Finally, having fixed $\lambda \in \mathcal{O}_{K}$ satisfying (2-1) and (2-2), we can still multiply $u$ by a unit so as to have, for instance:

$$
\begin{aligned}
& \sqrt{\varepsilon_{0}^{-1}\left|\mathrm{~N}_{K / \mathbb{Q}}(\tilde{x} / \lambda)\right|} \leq\left|\frac{\tilde{x}^{(1)}}{\lambda^{(1)}}\right|<\sqrt{\varepsilon_{0}\left|\mathrm{~N}_{K / \mathbb{Q}}(\tilde{x} / \lambda)\right|}, \\
& \sqrt{\varepsilon_{0}^{-1}\left|\mathrm{~N}_{K / \mathbb{Q}}(\tilde{x} / \lambda)\right|}<\left|\frac{\tilde{x}^{(2)}}{\lambda^{(2)}}\right| \leq \sqrt{\varepsilon_{0}\left|\mathrm{~N}_{K / \mathbb{Q}}(\tilde{x} / \lambda)\right|} .
\end{aligned}
$$

Using Lemma 2.3, we infer that

$$
\left|\mathrm{N}_{K / \mathbb{Q}}(\tilde{x} / \lambda)\right| \leq \sqrt{c_{1}^{\prime} c_{2}^{\prime}} \gamma_{K, 2}=\gamma_{K, 2}
$$

and that

$$
\left.\begin{array}{rl}
\left|\mathrm{N}_{K / \mathbb{Q}}(\tilde{y} / \lambda)\right| & \leq \gamma_{K, 2}, \\
\left|\frac{\tilde{x}^{(1)}}{\lambda^{(1)}} \frac{\tilde{y}^{(2)}}{\lambda^{(2)}}\right| & \leq \sqrt{c_{1}^{\prime}} \gamma_{K, 2}<\sqrt{\varepsilon_{0}} \gamma_{K, 2}, \\
\left|\frac{\tilde{x}^{(2)}}{\lambda^{(2)}} \frac{\tilde{y}^{(1)}}{\lambda^{(1)}}\right| & \leq \sqrt{c_{2}^{\prime}} \gamma_{K, 2} \leq \sqrt{\varepsilon_{0}} \gamma_{K, 2} .
\end{array}\right\}
$$

Clearly, $\lambda$ being fixed, the set of $\tilde{x} \in \mathcal{O}_{K}$ satisfying $(2-4)$ and $(2-3)$ is finite, and then, inequalities $(2-5)$ allow only a finite set of values for $\tilde{y}$. Going back to $(2-1)$, we see that

$$
\left|y^{(i)}\right|=\frac{\left|\tilde{y}^{(i)}\right|}{\left|\lambda^{(i)}\right|}\left|y_{2}^{(i)}\right|
$$

is bounded, and that

$$
\left|x^{(i)}\right|=\left|\frac{\tilde{x}^{(i)}}{\lambda^{(i)}}+\frac{\tilde{y}^{(i)}}{\lambda^{(i)}} x_{2}^{(i)}\right| \leq \frac{\left|\tilde{x}^{(i)}\right|}{\left|\lambda^{(i)}\right|}+C_{K} \frac{\left|\tilde{y}^{(i)}\right|}{\left|\lambda^{(i)}\right|}\left|y_{2}^{(i)}\right| .
$$

The explicit bounds of the lemma are then easily deduced from the various inequalities we have established.

In some particular instances, the preceding lemma can be restated in a simpler way. We say that $S$ admits a unimodular pair of minimal vectors if there exist $u, v \in M(S)$ such that $\mathcal{O}_{K} u \oplus \mathcal{O}_{K} v=\mathcal{O}_{K}^{2}$. Then:

Lemma 2.6. If $h(K)=1$, any Humbert form admitting a unimodular pair of minimal vectors is equivalent, up to scaling and integral equivalence, to a form

$$
S=\left(\left(\begin{array}{ll}
1 & b_{1} \\
b_{1} & c
\end{array}\right),\left(\begin{array}{ll}
1 & b_{2} \\
b_{2} & c^{-1}
\end{array}\right)\right)
$$

with $\varepsilon_{0}^{-1} \leq c<\varepsilon_{0}$. Moreover, $M(S) \supset\left\{e_{1}, e_{2}\right\}$ and any other minimal vector admits a representative $u=(x, y)^{t}$ modulo units satisfying

$$
\left|\mathrm{N}_{K / \mathbb{Q}}(x)\right| \leq \gamma_{K, 2}, \quad\left|\mathrm{~N}_{K / \mathbb{Q}}(y)\right| \leq \gamma_{K, 2}
$$

and

$$
\left|y^{(1)}\right|<\frac{\sqrt{\varepsilon_{0}} \gamma_{K, 2}}{\left|x^{(2)}\right|}, \quad\left|y^{(2)}\right| \leq \frac{\sqrt{\varepsilon_{0}} \gamma_{K, 2}}{\left|x^{(1)}\right|} .
$$

Proof. The assumption that $S$ admits a unimodular pair allows to take $u_{2}=e_{2}$ and $\lambda=1$ in the proof of Lemma 2.5. The conclusion follows. 
Having explicitly described the set $M_{K}$, we can pass on to step 2 of the algorithm, that is solve the systems

$$
S\left[u_{1}\right]=\cdots=S\left[u_{5}\right]=1,
$$

for all 5 -sets $T=\left\{u_{1}, \ldots, u_{5}\right\}$ in $M_{K}$ subject to the condition that any pair of vectors in $T$ satisfies Lemma 2.4. According to Lemma 2.5, we can assume that $u_{1}=e_{1}$, and write

$$
S=\left(\left(\begin{array}{ll}
1 & b_{1} \\
b_{1} & c_{1}
\end{array}\right),\left(\begin{array}{ll}
1 & b_{2} \\
b_{2} & c_{2}
\end{array}\right)\right)
$$

So $(2-7)$ is a system of polynomial equations in the 4 variables $b_{1}, c_{1}, b_{2}, c_{2}$ that we can solve using elimination theory (see the examples in the next section). When the condition of Lemma 2.6 is satisfied, we can assume that $u_{2}=e_{2}$ and the number of variables reduces to three $\left(c_{1}=c_{2}^{-1}\right)$. Finally, since minimal vectors are defined up to multiplication by units, and $M(S[U])=U^{-1} M(S)$ for any $U \in \mathrm{GL}\left(2, \mathcal{O}_{K}\right)$, we need to consider these different 5 -sets in $M_{K}$ only up to the following equivalence relation:

Definition 2.7. We call two 5 -sets $\left\{u_{1}, \ldots, u_{5}\right\}$ and $\left\{v_{1}, \ldots, v_{5}\right\}$ of $\mathcal{O}_{K}^{2}$ equivalent if there exists $U \in$ $\mathrm{GL}\left(2, \mathcal{O}_{K}\right)$ and $\left(\varepsilon_{1}, \ldots, \varepsilon_{5}\right) \in U_{K}^{5}$ such that

$$
U u_{i}=\varepsilon_{i} v_{i}, \quad i=1, \ldots, 5 .
$$

This remark shortens notably the computations in the next section.

\section{EXAMPLES}

Let $K=\mathbb{Q}(\sqrt{d})$, with $d>0$ a square free rational integer, and suppose that $h_{K}=1$. As usual, we identify $K$ with a subfield of $\mathbb{R}$, i.e., we fix an embedding of $K$, and we denote by $x^{\prime}$ the image of $x \in K$ by the nontrivial element of $\mathrm{Gal}_{K / \mathbb{Q}}$.

As mentioned above, the computations are much more easy if one can restrict to forms admitting a unimodular pair of minimal vectors. It turns out that, for some small discriminants, one can show $a$ priori that this condition will always hold for Humbert forms with sufficiently many minimal vectors for example, perfect forms. This is based on observations of the following kind:
Lemma 3.1. Let $S \in P_{2, K}$ and $v_{i}=\left(\alpha_{i}, \beta_{i}\right)^{t} \in M(S)$ for $1 \leq i \leq s$, and let $v_{i, j}$, for $1 \leq i \neq j \leq s$, be the determinants of the corresponding pairs:

$$
v_{i, j}=\operatorname{det}\left(\begin{array}{cc}
\alpha_{i} & \alpha_{j} \\
\beta_{i} & \beta_{j}
\end{array}\right) .
$$

Then, for a fixed prime ideal $\mathfrak{p}$, with corresponding valuation $v_{\mathfrak{p}}$, we have: If $\{i, j, k\} \subset\{1, \ldots, s\}$ is ordered so that $v_{\mathfrak{p}}\left(v_{i, j}\right) \geq \max \left(v_{\mathfrak{p}}\left(v_{\imath, k}\right), v_{\mathfrak{p}}\left(v_{j, k}\right)\right)$, then

$$
v_{\mathfrak{p}}\left(v_{i, j}\right) \geq v_{\mathfrak{p}}\left(v_{i, k}\right)=v_{\mathfrak{p}}\left(v_{j, k}\right) .
$$

In particular, if $\{i, j\}$ is such that $v_{\mathfrak{p}}\left(v_{i, j}\right)$ is maximal among all pairs $\{i, j\}$, we have

$$
v_{\mathfrak{p}}\left(v_{i, j}\right) \geq v_{\mathfrak{p}}\left(v_{i, k}\right)=v_{\mathfrak{p}}\left(v_{j, k}\right) \quad \text { for } k \neq i, j .
$$

Proof. Expressing $v_{k}$ as a linear combination, with coefficients in $K$, of $v_{i}$ and $v_{j}$, we get

$$
\begin{aligned}
v_{k} & =\left(\alpha_{k}, \beta_{k}\right)=\frac{v_{k, i}}{v_{i, j}} v_{i}+\frac{v_{k, j}}{v_{j, i}} v_{j} \\
& =\left(\frac{v_{k, i} \alpha_{i}-v_{k, j} \alpha_{j}}{v_{i, j}}, \frac{v_{k, i} \beta_{i}-v_{k, j} \beta_{j}}{v_{i, j}}\right),
\end{aligned}
$$

and similarly permuting $i, j$ and $k$. If $v_{\mathfrak{p}}\left(v_{i, j}\right)$ were strictly less than both $v_{\mathfrak{p}}\left(v_{i, k}\right)$ and $v_{\mathfrak{p}}\left(v_{j, k}\right)$, then the valuation of $\alpha_{k}$ and $\beta_{k}$ would be strictly positive, contradicting the primitivity of $v_{k}$. Thus

$$
v_{\mathfrak{p}}\left(v_{i, j}\right) \geq \min \left(v_{\mathfrak{p}}\left(v_{i, k}\right), v_{\mathfrak{p}}\left(v_{j, k}\right)\right),
$$

and this holds for any permutation of $i, j, k$. This is easily seen to imply the assertion of the lemma.

3A. $K=\mathbb{Q}(\sqrt{5})$

Beside Cohn's general upper bound $\gamma_{K, 2}<d_{K} / 2=$ 2.5 , we can use in this case Götzky's estimate [1928]

$$
\gamma_{\mathbb{Q}(\sqrt{5}), 2} \leq \frac{16}{-9+\sqrt{312}}<2
$$

Then Lemma 2.4 implies that any two noncolinear minimal vectors of $S \in \mathbf{P}$ generate $\mathcal{O}_{K}^{2}$, so Lemma 2.6 applies to any Humbert form $S$ with $\# M(S) \geq$ 2 , and in particular to any perfect Humbert form. So we can restrict to forms

$$
S=\left(\left(\begin{array}{ll}
1 & b_{1} \\
b_{1} & c
\end{array}\right),\left(\begin{array}{ll}
1 & b_{2} \\
b_{2} & c^{-1}
\end{array}\right)\right)
$$

with $m(S)=1$. The right-hand side of $(2-6)$ is less than 2, and for the same reason as before, this implies that the coordinates of minimal vectors distinct from $e_{1}$ and $e_{2}$ are units. Up to multiplication by a suitable unit, we can assume that these 
vectors are of the shape $(\alpha, 1)^{t}$, with $\alpha \in U_{K}$, and Lemma 2.6 provides explicit bounds for $\alpha$, namely $\alpha \in\left\{ \pm 1, \pm \tau, \pm \tau^{\prime}\right\}$, where $\tau=\frac{1}{2}(1+\sqrt{5})$. Finally, taking into account that each pair of minimal vectors has to satisfy Lemma 2.4, it is easily seen that, up to equivalence in the sense of Definition 2.7, the only 5 -set to consider is

$$
T=\left\{(1,0)^{t},(0,1)^{t},(1,1)^{t},(\tau, 1)^{t},\left(-\tau^{\prime}, 1\right)^{t}\right\} .
$$

The requirement that vectors $u \in T$ satisfy $S(u)=1$ amounts to requiring that the following polynomials in $c, b_{1}$ and $b_{2}$ vanish simultaneously:

$$
\begin{aligned}
& \left(1+2 b_{2}\right) c^{2}+\left(1+2 b_{2}\right)\left(1+2 b_{1}\right) c+\left(1+2 b_{1}\right), \\
& \tau^{\prime}\left(\tau^{\prime}+2 b_{2}\right) c^{2}-\left(\tau^{\prime}+2 b_{2}\right)\left(\tau+2 b_{1}\right) c+\tau\left(\tau+2 b_{1}\right), \\
& \tau\left(-\tau+2 b_{2}\right) c^{2}+\left(-\tau+2 b_{2}\right)\left(-\tau^{\prime}+2 b_{1}\right) c+\tau^{\prime}\left(-\tau^{\prime}+2 b_{1}\right) .
\end{aligned}
$$

Eliminating $b_{2}$ between $(3-1)=0$ and $(3-2)=0$ yields

$$
\begin{array}{r}
\left(8 \tau c^{2}+(8+8 \tau) c\right) b_{1}^{2}+\left((4+4 \tau) c^{3}+16 \tau c^{2}+(8+12 \tau) c\right) b_{1} \\
+\left(2 c^{4}+(4+2 \tau) c^{3}+(2+6 \tau) c^{2}+(2+4 \tau) c\right)=0
\end{array}
$$

and doing the same between $(3-1)=0$ and $(3-3)=$ 0 yields

$$
\begin{gathered}
\left((8+8 \tau) c^{2}+8 \tau c\right) b_{1}^{2}+\left((8+12 \tau) c^{3}+16 \tau c^{2}+(4+4 \tau) c\right) b_{1} \\
\quad+\left((2+4 \tau) c^{4}+(2+6 \tau) c^{3}+(4+2 \tau) c^{2}+2 c\right)=0 .
\end{gathered}
$$

Finally, eliminating $b_{1}$ between these two equations, we find that $c$ has to satisfy

$$
c^{5}(c-1)^{2}\left(c-\tau^{4}\right)\left(c-\tau^{4}\right)\left(c^{2}+3 c+1\right)=0,
$$

whence $c=1, \tau^{2}$ or $\tau^{\prime 2}$. If one substitutes the value $c=1$ into (3-2) and (3-3), the two equations are easily seen to be equivalent, so the initial system eventually reduces to the system

$$
\begin{aligned}
\left(1+2 b_{2}\right)+\left(1+2 b_{2}\right)\left(1+2 b_{1}\right)+\left(1+2 b_{1}\right) & =0, \\
\tau^{\prime}\left(\tau^{\prime}+2 b_{2}\right)-\left(\tau^{\prime}+2 b_{2}\right)\left(\tau+2 b_{1}\right)+\tau\left(\tau+2 b_{1}\right) & =0 .
\end{aligned}
$$

Using the conditions $1-b_{i}^{2}>0\left(A_{i}>0\right)$, calculation yields $\left(b_{1}, b_{2}\right)=\left(-\frac{1}{2} \tau,-\frac{1}{2} \tau^{\prime}\right)$ or $\left(\frac{1}{2} \tau^{\prime}, \frac{1}{2} \tau\right)$. It is then easily checked that substituting $c=\tau^{2}$ or $\tau^{\prime 2}$ in (3-1)-(3-3) leads to equivalent solutions modulo integral equivalence. Thus, we have shown that the only possible perfect binary forms over $\mathbb{Q}(\sqrt{5})$ are

$$
\mathcal{S}=\left(\left(\begin{array}{cc}
1 & -\frac{1}{2} \tau \\
-\frac{1}{2} \tau & 1
\end{array}\right),\left(\begin{array}{cc}
1 & -\frac{1}{2} \tau^{\prime} \\
-\frac{1}{2} \tau^{\prime} & 1
\end{array}\right)\right)
$$

and

$$
\mathcal{S}^{\prime}=\left(\left(\begin{array}{cc}
1 & \frac{1}{2} \tau^{\prime} \\
\frac{1}{2} \tau^{\prime} & 1
\end{array}\right),\left(\begin{array}{cc}
1 & \frac{1}{2} \tau \\
\frac{1}{2} \tau & 1
\end{array}\right)\right)
$$

But the two are equivalent:

$$
\mathcal{S}^{\prime}=\tau^{-2} \cdot \mathcal{S}\left[\left(\begin{array}{cc}
-1+\tau & -1+\tau \\
0 & 1
\end{array}\right)\right] .
$$

Since we know from [Coulangeon 2001] that there always exists at least one perfect form and at least one eutactic form, we conclude that $\mathcal{S}$ is perfect and eutactic. This can of course also be checked using the original definitions. Thus:

Theorem 3.2. Up to scaling and equivalence under $\mathrm{GL}\left(2, \mathcal{O}_{K}\right)$, the form $\mathcal{S}$ is the only binary perfect Humbert form over $\mathbb{Q}(\sqrt{5})$. It has $\# M(\mathcal{S})=5$ minimal vectors, and is moreover eutactic, hence extreme. Consequently

$$
\gamma_{\mathbb{Q}(\sqrt{5}), 2}=\gamma(\mathcal{S})=\frac{4}{\sqrt{5}} .
$$

3B. $K=\mathbb{Q}(\sqrt{2})$

In this case we can again apply Lemma 2.6, thanks to the following lemma:

Lemma 3.3. Any binary Humbert form $S$ over $\mathbb{Q}(\sqrt{2})$ with $\# M(S) \geq 3$ admits a unimodular pair.

Proof. We let $\# M(S)=\left\{v_{1}, \ldots, v_{s}\right\}, s \geq 3$, and use the notation $v_{i, j}$ for the determinants of the various pairs, as in Lemma 3.1. Assume that no pair is unimodular, i.e., none of the $v_{i, j}$ is a unit. From Lemma 2.4, using the bound $\gamma_{K, 2}<\left|d_{K}\right| / 2=4$, we see that the only possible prime divisor for $v_{i, j}$ is $\mathfrak{p}=\sqrt{2} \mathcal{O}_{K}$, and that all $v_{i, j}$ must satisfy $v_{\mathfrak{p}}\left(v_{i, j}\right)=$ 1. Without loss of generality, we can assume that $v_{1}=(1,0)^{t}$. Writing $v_{i}=\left(\alpha_{i}, \beta_{i}\right)^{t}$, and multiplying each $v_{i}$ by a suitable unit, we can therefore assume that $\beta_{i}=\sqrt{2}$ for $2 \leq i \leq s$. The $v_{i}$ being primitive, we moreover have that $v_{\mathfrak{p}}\left(\alpha_{i}\right)=0,2 \leq i \leq s$. Now the condition $v_{\mathfrak{p}}\left(v_{i, j}\right)=1$, for $2 \leq i<j \leq s$ reads $v_{\mathfrak{p}}\left(\alpha_{i}-\alpha_{j}\right)=0=v_{\mathfrak{p}}\left(\alpha_{i}\right)=v_{\mathfrak{p}}\left(\alpha_{j}\right)$, which is impossible $\left(\mathcal{O}_{K} / \mathfrak{p} \simeq \mathbb{F}_{2}\right)$. So at least one $v_{i, j}$ is a unit.

We used PARI to classify all 5 -sets of vectors satisfying Lemmas 2.3, 2.4 and 2.6 according to the equivalence relation of Definition 2.7. There are two 
inequivalent sets to consider:

$T_{1}=\left\{(1,0)^{t},(0,1)^{t},(-1-\sqrt{2}, 1)^{t},(-\sqrt{2}, 1)^{t},(-1,1)^{t}\right\}$, $T_{2}=\left\{(1,0)^{t},(0,1)^{t},(-1-\sqrt{2}, 1)^{t},(-\sqrt{2}, 1)^{t},(1,1)^{t}\right\}$.

They correspond each to a system of polynomial equations, analogous to the system of the previous section given by the vanishing of $(3-1)-(3-3)$. As before, we eliminate successively $b_{2}$ and $b_{1}$ and factorize over $K$ the resulting polynomial in $c$, to find that $c$ has to satisfy

$$
\begin{aligned}
\left(c^{2}-(2+\sqrt{2}) c+3\right. & +2 \sqrt{2}) \\
& \times\left(c^{2}-(1+\sqrt{2}) c-(3+2 \sqrt{2})\right) \\
& \times\left(c^{2}-\sqrt{2} c-1\right)=0
\end{aligned}
$$

in the case of $T_{1}$, or

$$
\begin{aligned}
&\left(c^{4}-(10+\right.4 \sqrt{2}) c^{3}+(4+4 \sqrt{2}) c^{2} \\
&+(14+8 \sqrt{2}) c-(3+2 \sqrt{2})) \\
& \times\left(c^{2}+(1+\sqrt{2}) c-(3+2 \sqrt{2})\right)=0 .
\end{aligned}
$$

in the case of $T_{2}$.

One can compute the real positive roots of these equations, substitute these values of $c$ in the initial system, and then solve the corresponding systems in $b_{1}$ and $b_{2}$. Afterwards, one has to check that the resulting form are positive definite, and that their minimum is 1 . In the case of $T_{1}$ and equation (3-5), the only root that leads to a Humbert form is $\frac{\sqrt{6}+\sqrt{2}}{2}$, the positive root of $c^{2}-\sqrt{2} c-1$, and the corresponding form is

$$
\mathcal{S}_{1}=\left(\left(\begin{array}{cc}
1 & \frac{1+\sqrt{2}}{2} \\
\frac{1+\sqrt{2}}{2} & \frac{\sqrt{6}+\sqrt{2}}{2}
\end{array}\right),\left(\begin{array}{cc}
1 & \frac{1-\sqrt{2}}{2} \\
\frac{1-\sqrt{2}}{2} & \frac{\sqrt{6}-\sqrt{2}}{2}
\end{array}\right)\right) .
$$

In the case of $T_{2}$ and equation (3-6), the only root that leads to a Humbert form is $\frac{1+\sqrt{2}}{2}(\sqrt{5}-1$ ) (positive root of $\left.c^{2}+(1+\sqrt{2}) c-(3+2 \sqrt{2})\right)$, the corresponding form being

$\mathcal{S}_{2}=\left(\left(\begin{array}{cc}1 & \frac{\sqrt{2}}{2} \\ \frac{\sqrt{2}}{2} & \frac{(1+\sqrt{2})(\sqrt{5}-1)}{2}\end{array}\right),\left(\begin{array}{cc}1 & \frac{-\sqrt{2}}{2} \\ \frac{-\sqrt{2}}{2} & \frac{(1-\sqrt{2})(\sqrt{5}+1)}{2}\end{array}\right)\right)$.

But it is readily checked that $m\left(\mathcal{S}_{2}\right)<1$; for instance, $\mathcal{S}_{2}([2+\sqrt{2},-1-\sqrt{2}])=7-2 \sqrt{10}<1$. Thus:

Theorem 3.4. Up to scaling and equivalence under $\mathrm{GL}\left(2, \mathcal{O}_{K}\right)$, the form $\mathcal{S}_{1}$ is the only binary perfect Humbert form over $\mathbb{Q}(\sqrt{2})$. It has $\# M\left(\mathcal{S}_{1}\right)=5$ minimal vectors, and is eutactic, hence extreme. Consequently

$$
\gamma_{\mathbb{Q}(\sqrt{2}), 2}=\gamma\left(\mathcal{S}_{1}\right)=\frac{4}{2 \sqrt{6}-3} .
$$

3C. $\mathrm{K}=\mathbb{Q}(\sqrt{3})$

Lemma 3.5. Any binary Humbert form $S$ over $\mathbb{Q}(\sqrt{3})$ with $\# M(S) \geq 5$ admits a unimodular pair.

Proof. We use the same notation as in the previous subsection and assume that none of the $v_{i, j}$ is a unit. Thanks to Lemma 2.4 and to the bound

$$
\gamma_{K, 2}<\frac{1}{2}\left|d_{K}\right|=6
$$

the only possible prime divisors for the $v_{i, j}$ are $\mathfrak{p}=$ $\sqrt{3} \mathcal{O}_{K}$ and $\mathfrak{q}=(1-\sqrt{3}) \mathcal{O}_{K}$. Moreover, a given $v_{i, j}$ cannot be divisible by both $\mathfrak{p}$ and $\mathfrak{q}$, since

$$
\left|\mathrm{N}_{K / \mathbb{Q}}(\mathfrak{p q})\right|=6>\gamma_{K, 2} \text {. }
$$

We claim that $\mathfrak{p}$ or $\mathfrak{q}$ has to divide all $v_{i, j}$ simultaneously. Suppose, for instance, that $\mathfrak{p}$ divides $v_{i, j}$. By Lemma 3.1, for any $k \neq i, j$, either $\mathfrak{p}$ divides both $v_{i, k}$ and $v_{j, k}$, or both have valuation 0 . But in the second case, $v_{i, k}$ and $v_{j, k}$ would be divisible by $\mathfrak{q}$, and so would $v_{i, j}$, by Lemma 3.1 again. So $\mathfrak{p q}$ would divide $v_{i, j}$, which is impossible. The same holds replacing $\mathfrak{p}$ by $\mathfrak{q}$, which proves the claim. Let $m=\max _{i<j} v_{\mathfrak{q}}\left(v_{i, j}\right)$. From Lemma 2.4 and bound (3-7), one has $m \leq 2$. If $m=0$, then all $v_{i, j}$ are divisible by $\mathfrak{p}$, with valuation 1 , and we easily derive a contradiction, arguing exactly as in Lemma 3.3. So we assume that $m \geq 1$. Due to the previous remarks, this implies that all $v_{i, j}$ are divisible by $\mathfrak{q}$. We claim that for a given $i$, and for $j \neq k$ one cannot have $v_{\mathfrak{q}}\left(v_{i, j}\right)=v_{\mathfrak{q}}\left(v_{i, k}\right)=2$. Indeed, if such a triple $\{i, j, k\}$ existed, we could assume, up to a change of basis, that $v_{i}=(1,0)^{t}$ (since $v_{i}$ is primitive), $v_{j}=$ $\left(\alpha_{j}, \beta_{j}\right)^{t}, v_{k}=\left(\alpha_{k}, \beta_{k}\right)^{t}$, with $v_{\mathfrak{q}}\left(\beta_{j}\right)=v_{\mathfrak{q}}\left(\beta_{k}\right)=2$, and $v_{\mathfrak{q}}\left(\alpha_{j}\right)=v_{\mathfrak{q}}\left(\alpha_{k}\right)=0$ (primitivity of $v_{j}$ and $\left.v_{k}\right)$. Scaling $v_{j}$ and $v_{k}$ by suitable units, we can moreover assume that $\beta_{j}=\beta_{k}$. Now the condition $v_{\mathfrak{p}}\left(v_{j, k}\right) \leq 2$, implies $v_{\mathfrak{p}}\left(\alpha_{k}-\alpha_{j}\right)=0$, which is impossible $\left(\mathcal{O}_{K} / \mathfrak{q} \simeq \mathbb{F}_{2}\right)$. Finally, assume, without loss of generality, that $v_{1}=(1,0)^{t}$. The previous observation, together with the assumption that $\mathfrak{q}$ divides all the $v_{i, j}$, and the fact that $\# M(S) \geq 5$, imply that $v_{\mathfrak{q}}\left(v_{1, j}\right)=v_{\mathfrak{q}}\left(v_{1, k}\right)=v_{\mathfrak{q}}\left(v_{1, l}\right)=1$ for at least three distinct integers $j, k, l$, and that one of the pairs, say 
$\{j, k\}$, satisfies $v_{\mathfrak{q}}\left(v_{j, k}\right)=1$. Using the coordinates of $v_{j}$ and $v_{k}$, scaled by suitable units, we conclude again that $v_{\mathfrak{q}}\left(\alpha_{k}\right)=v_{\mathfrak{q}}\left(\alpha_{j}\right)=v_{\mathfrak{q}}\left(\alpha_{k}-\alpha_{j}\right)=0$, a contradiction.

Here the classification of 5 -sets of vectors satisfying Lemma $2.3,2.4$ and 2.6 is considerably more complicated. There are 37 such sets to consider, up to the equivalence relation of Definition 2.7. We solved the corresponding systems in the same way as in the previous subsections. Only 24 of them lead to actual Humbert forms i.e., positive definite. They fall into 2 distinct classes modulo integral equivalence and scaling, among which only one has minimum 1. This is obtained with the set

$$
\begin{aligned}
T_{1}^{\prime}=\left\{(1,0)^{t},(0,1)^{t},\right. & (-u, \sqrt{3})^{t}, \\
& \left.(-1-\sqrt{3}, \sqrt{3})^{t},(-u, 1+\sqrt{3})^{t}\right\},
\end{aligned}
$$

where $u=2+\sqrt{3}$ is the fundamental unit of $\mathbb{Q}(\sqrt{3})$. The corresponding form is

$$
\mathcal{S}_{1}^{\prime}=\left(\left(\begin{array}{cc}
1 & \frac{1}{2} u \\
\frac{1}{2} u & u
\end{array}\right),\left(\begin{array}{cc}
1 & \frac{1}{2} u^{\prime} \\
\frac{1}{2} u^{\prime} & u^{\prime}
\end{array}\right)\right)
$$

As in the case of $\mathbb{Q}(\sqrt{5})$, we can immediately conclude that it is both perfect and eutactic. It has $\# M\left(S_{1}^{\prime}\right)=12$ minimal vectors (up to units), given by

$$
\begin{array}{r}
M\left(S_{1}^{\prime}\right)=\left\{(0,1)^{t},(1,0)^{t},(-u, \sqrt{3})^{t},(-1-\sqrt{3}, \sqrt{3})^{t},\right. \\
(-3-2 \sqrt{3}, 1+\sqrt{3})^{t},(-u, 1+\sqrt{3})^{t},(-u, 1)^{t},(-2,1)^{t}, \\
\left.(-1-\sqrt{3}, 1)^{t},(-1,1)^{t},(-\sqrt{3}, 1)^{t},(-u, 2)^{t}\right\} .
\end{array}
$$

Thus:

Theorem 3.6. Up to scaling and equivalence under $\mathrm{GL}\left(2, \mathcal{O}_{K}\right)$, the form $\mathcal{S}_{1}^{\prime}$ is the only binary perfect
Humbert form over $\mathbb{Q}(\sqrt{3})$. It has $\# M\left(\mathcal{S}_{1}^{\prime}\right)=12$ minimal vectors, and is eutactic, hence extreme. Consequently

$$
\gamma_{\mathbb{Q}(\sqrt{3}), 2}=\gamma\left(\mathcal{S}_{1}^{\prime}\right)=4
$$

\section{REFERENCES}

[Baeza and Icaza 1997] R. Baeza and M. I. Icaza, "On Humbert-Minkowski's constant for a number field", Proc. Amer. Math. Soc. 125:11 (1997), 3195-3202.

[Cohn 1965a] H. Cohn, "A numerical survey of the floors of various Hilbert fundamental domains", Math. Comp. 19 (1965), 594-605.

[Cohn 1965b] H. Cohn, "On the shape of the fundamental domain of the Hilbert modular group", pp. 190202 in Theory of numbers, edited by A. L. Whiteman, Proc. Symp. Pure Math. 8, 1965.

[Coulangeon 2001] R. Coulangeon, "Voronoï theory over algebraic number fields", Monographies de l'Enseignement Mathématique 37 (2001), 147-162.

[Götzky 1928] F. Götzky, "Über eine zahlentheoretische Anwendung von Modulfunktionen zweier Veränderlichen", Math. Ann. 100 (1928), 411-437.

[Humbert 1940] P. Humbert, "Théorie de la réduction des formes quadratiques définies positives dans un corps algébrique $K$ fini", Comment. Math. Helv. 12 (1940), 263-306.

[Icaza 1997] M. I. Icaza, "Hermite constant and extreme forms for algebraic number fields", J. London Math. Soc. (2) 55:1 (1997), 11-22.

[Ohno and Watanabe 2001] S. Ohno and T. Watanabe, "Estimates of Hermite constants for algebraic number fields", Comment. Math. Univ. St. Paul. 50:1 (2001), $53-63$.

Ricardo Baeza, Instituto de Matemática y Física, Universidad de Talca, Casilla 721, Talca, Chile.

(rbaeza@inst-mat.utalca.cl)

Renaud Coulangeon, Laboratoire A2X, Université Bordeaux I, 351 cours de la Libération, FR-33405, Talence, France (renaud.coulangeon@math.u-bordeaux.fr)

Maria Ines Icaza, Icaza, Instituto de Matemática y Física, Universidad de Talca, Casilla 747, Talca, Chile (icaza@inst-mat.utalca.cl)

Manuel O'Ryan, Icaza, Instituto de Matemática y Física, Universidad de Talca, Casilla 747, Talca, Chile (moryan@inst-mat.utalca.cl) 
